\title{
Supporting InformationSequential Release Platform of Heparin and Urokinase with Dual Physical (NIR-II and Bubbles) Assistance for Deep Venous Thrombosis
}

Zhiwei Zhong, ${ }^{\dagger}$ Cuifu Fang,,$^{\dagger}$ Shasha He," Teng Zhang, ${ }^{\dagger}$ Shichen Liu, ${ }^{\dagger}$ Yini Zhang,,

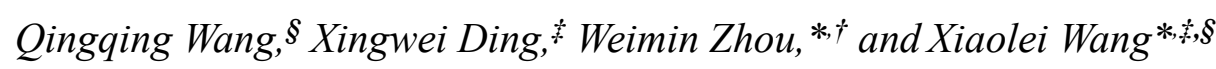

${ }^{\dagger}$ Department of Vascular Surgery, The Second Affiliated Hospital of Nanchang University, Nanchang, 330006, China.

National Engineering Research Center for Bioengineering Drugs and the Technologies, Institute of Translational Medicine, Nanchang University, Nanchang 330088, China.

$\S$ College of Chemistry, Nanchang University, Nanchang 330088, China.

"Department of Endocrinology, The First Affiliated Hospital of Nanchang University, Nanchang, 330006, China.

*Corresponding authors.

*E-mail:zwmsubmit@126.com

*E-mail: wangxiaolei@ncu.edu.cn

Number of pages: 15

Number of pictures: 17 
Number of tables:0

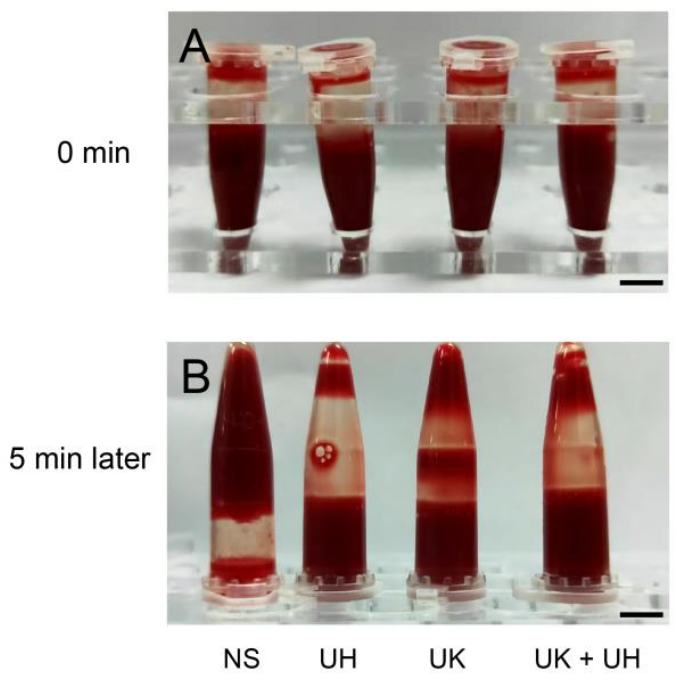

Figure S1. The photos of coagulation after different treatments (scale bar: $1 \mathrm{~cm}$ ).

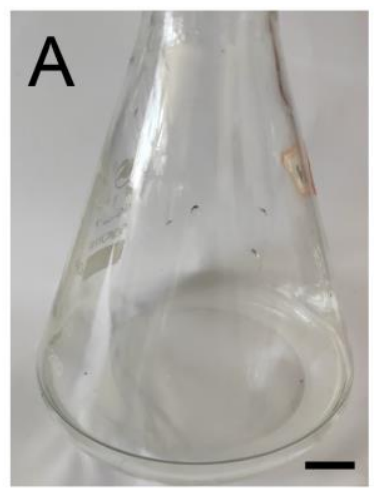

HMSNs

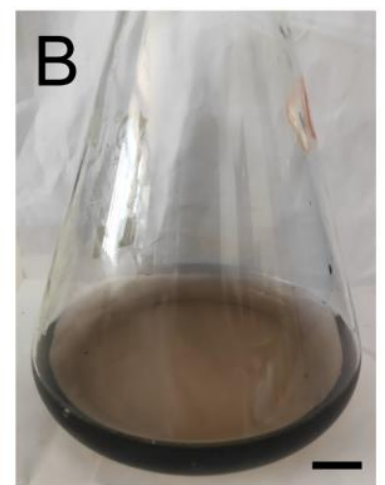

PDA@HMSNs

Figure S2. The photos of a pure aqueous solution of (A) HMSNs and (B) PDA@HMSNs (scale bar: 2 cm). 


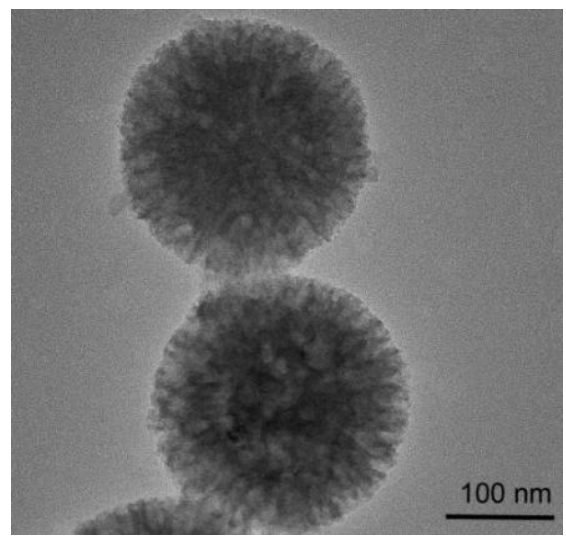

Figure S3. TEM images of HMSNs loaded with UK and LM.

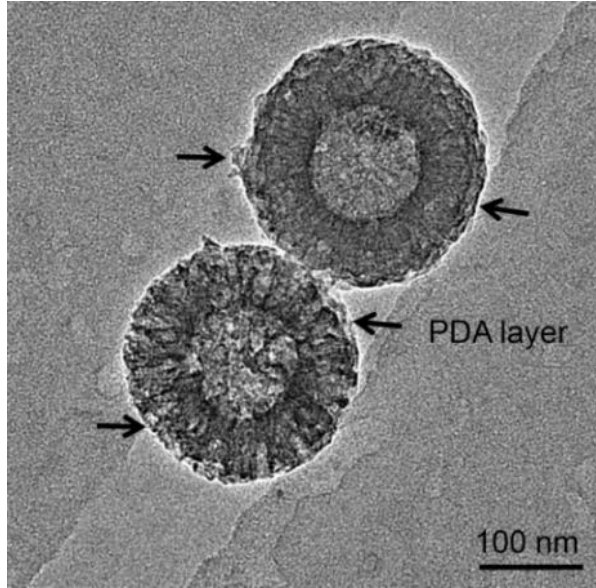

Figure S4. TEM images of PDA@HMSNs.
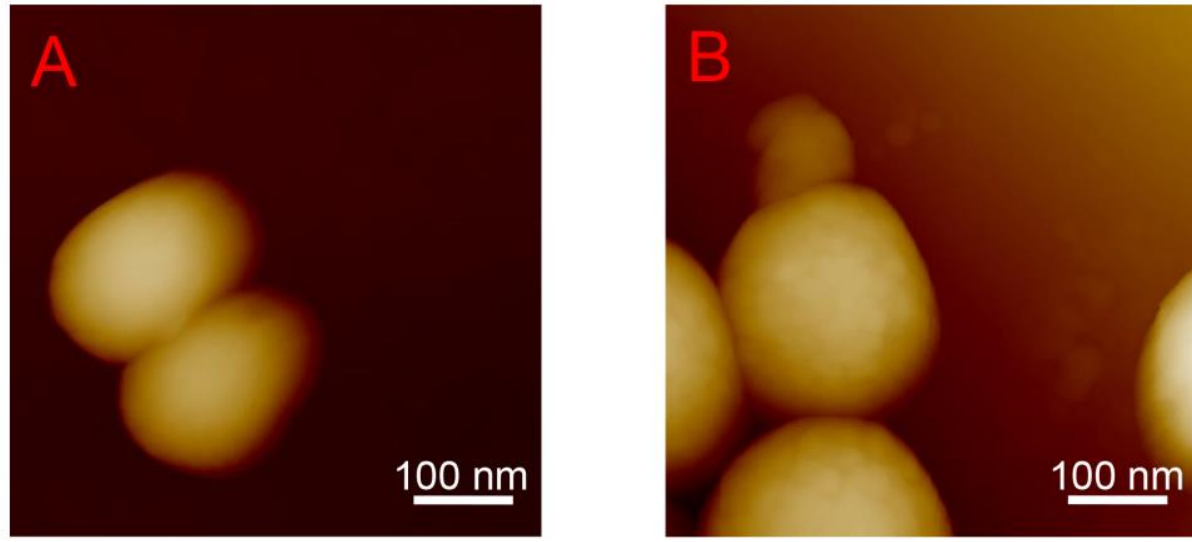

Figure S5. The AFM image of (A) HMSNs and (B) PDA@HMSNs. 


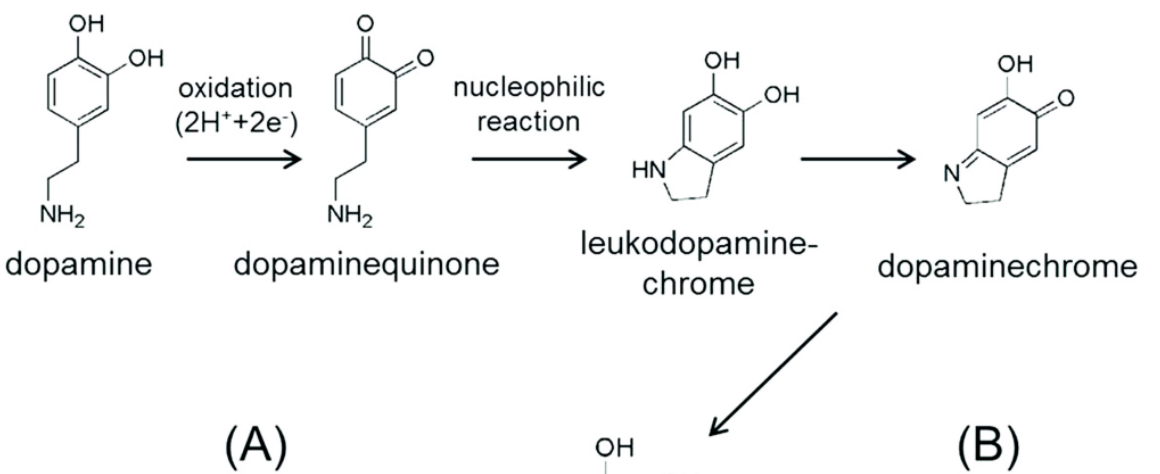

Covalent oxidative polymerization

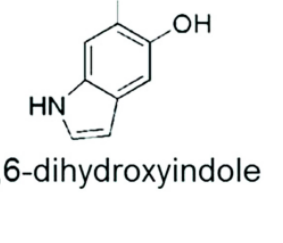

Physical self-assembly
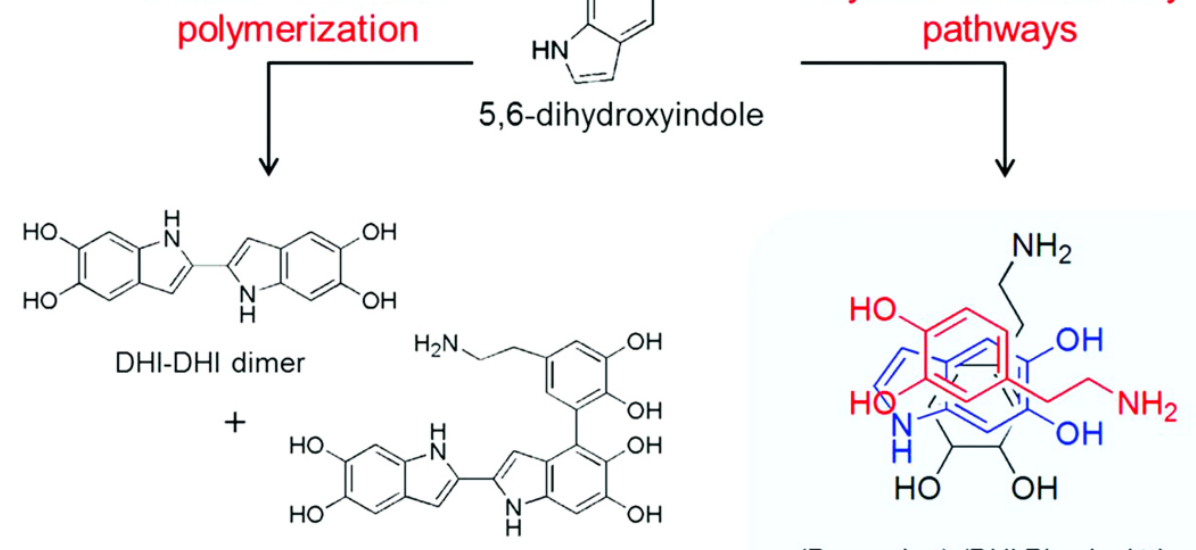

Dopamine-DHI-DHI trimeric conjugate

(Dopamine) $)_{2} / \mathrm{DHI}$ Physical trimer

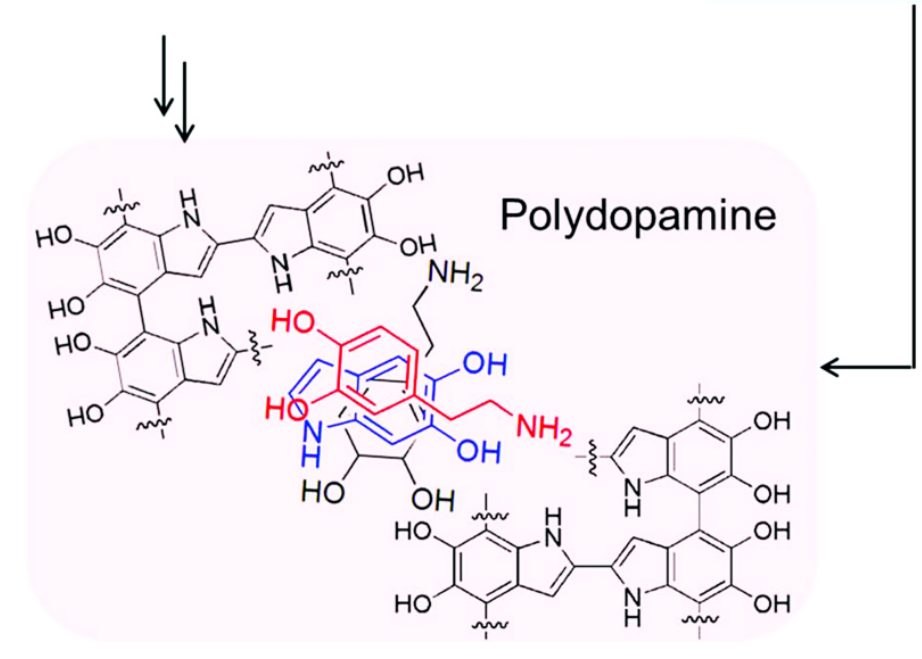

Figure S6. PDA synthesis pathways: (A) covalent bond-forming oxidative polymerization and (B) physical self-assembly of DA and DHI. Reproduced with permission from ref 1 . Copyright 2012 John Wiley \& Sons. ${ }^{[1]}$ 


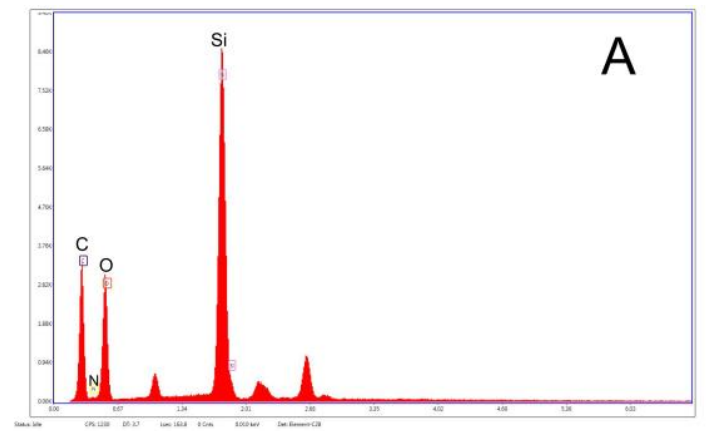

HMSNs

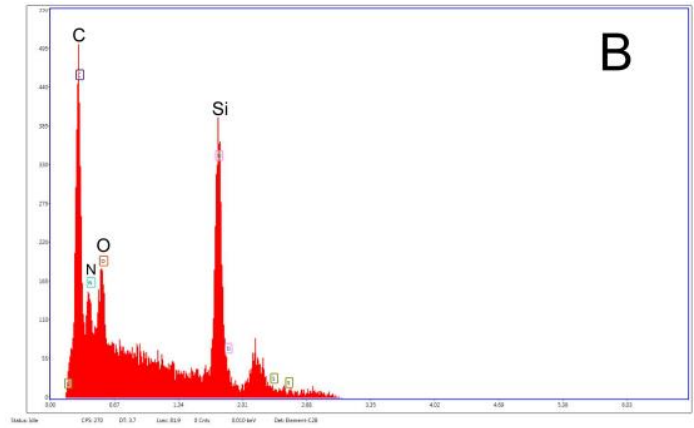

PDA@HMSNs

Figure S7. The EDS spectrum of (A) HMSNs and (B) PDA@HMSNs.

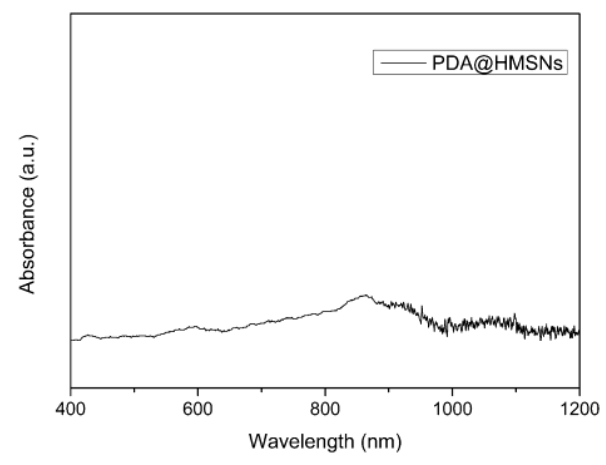

Figure S8. UV-vis-NIR absorption spectrum of PDA@HMSNs.

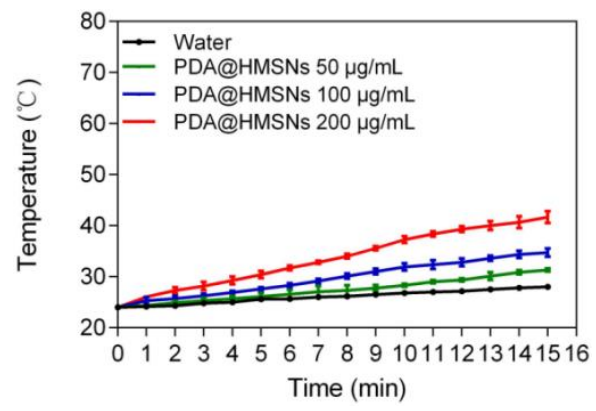

Figure S9. Temperature rise curve of different concentrations of PDA@HMSNs at $808 \mathrm{~nm}$ laser irradiation $\left(1.0 \mathrm{~W} \mathrm{~cm}^{-2}\right)$. 

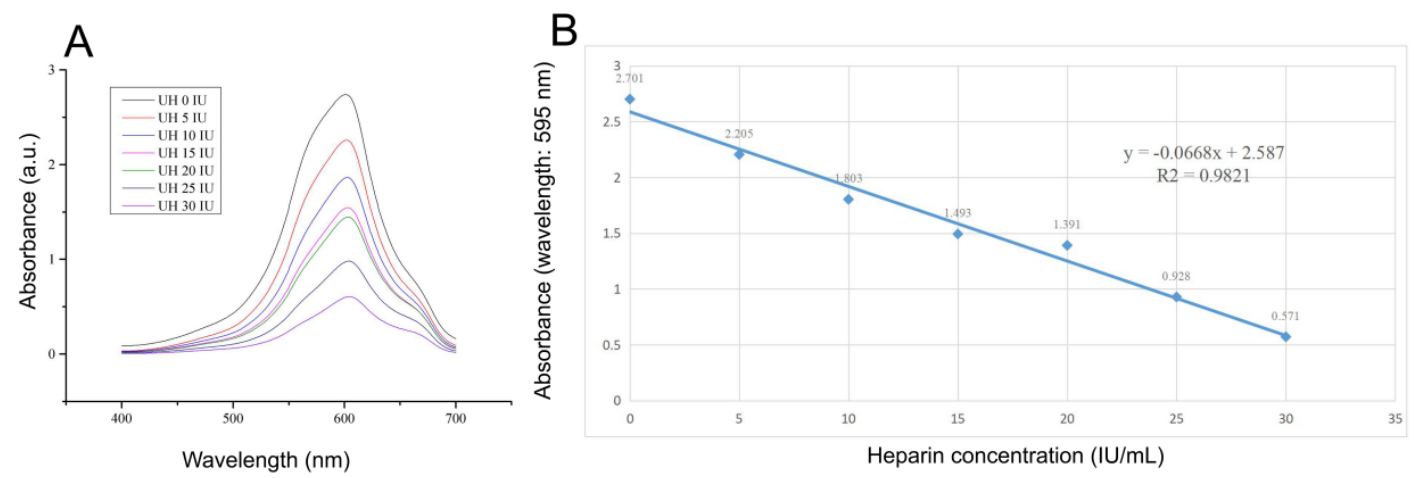

Figure S10. (A) Absorption spectra of azure A solution $(0.2 \%, \mathrm{w} / \mathrm{v})$ after the addition of different concentrations of UH. (B) Calibration curve of the concentration of UH and the value of absorbance.

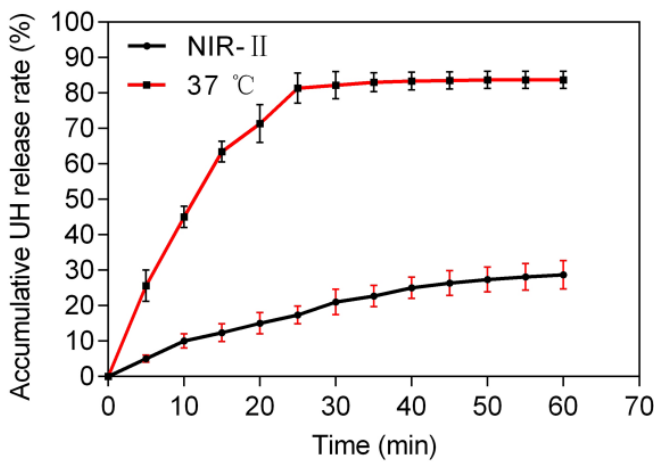

Figure S11. Release rate of UH from UK-UH@PDA@HMSNs under different conditions in vitro.

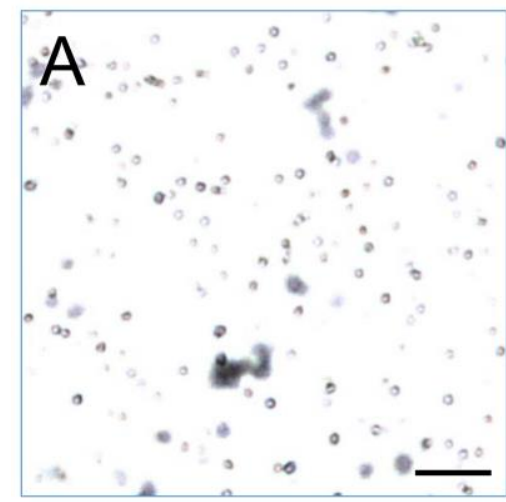

NIR-II $10 \mathrm{~min}$

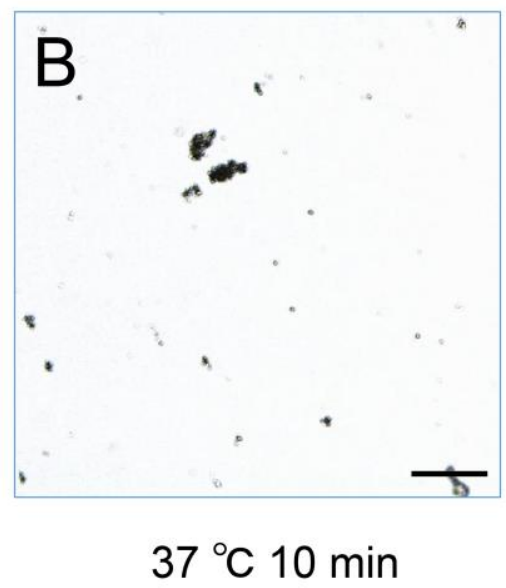

$37^{\circ} \mathrm{C} 10 \min$

Figure S12. (A) UK-UH@PDA@HMSNs was treated with NIR-II (1064 nm, 1.0 W 
$\mathrm{cm}^{-2}$ ). (B) UK-UH@PDA@HMSNs was treated with $37{ }^{\circ} \mathrm{C}$ (scale bar: $100 \mu \mathrm{m}$ ).

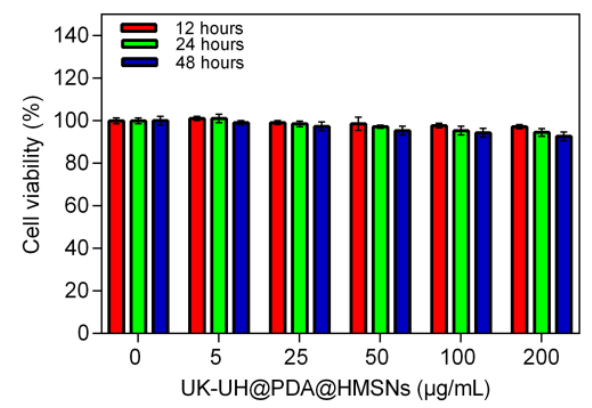

Figure S13. Cell viability of HUVECs treated with different concentrations of UK-UH@PDA@HMSNs.

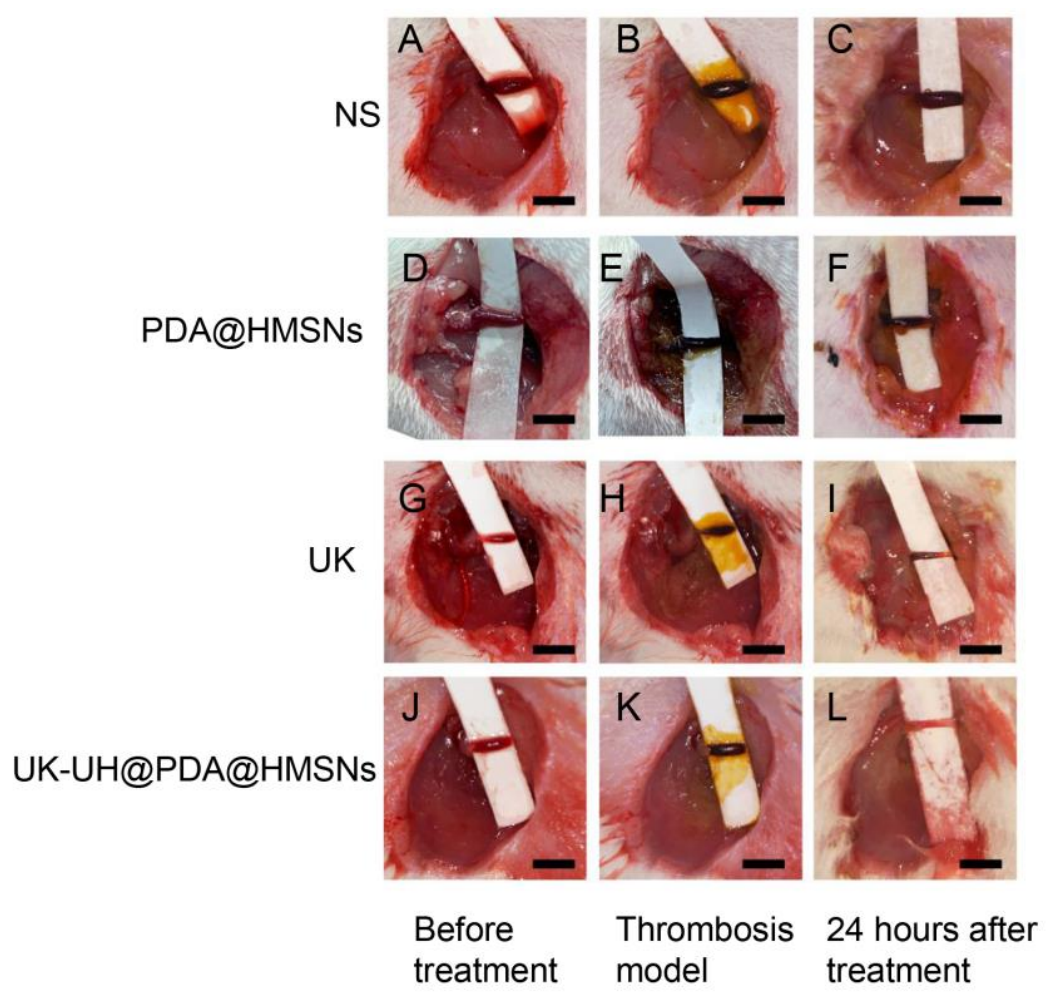

Figure S14. Photos (scale bar: $1 \mathrm{~cm}$ ) of clots treated with NS, PDA@HMSNs, UK, and UK-UH@PDA@HMSNs before and after NIR-II irradiation for 24 hours. 


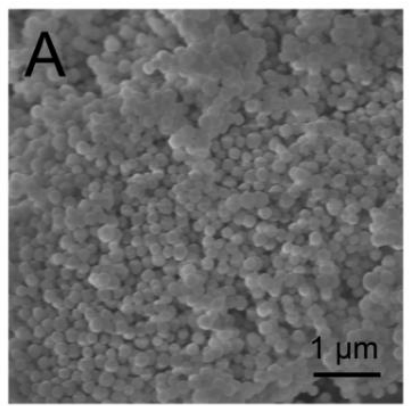

Day 0

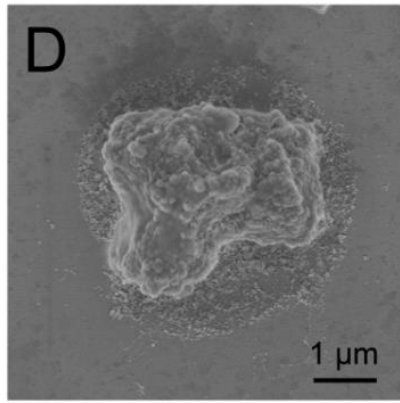

Day 5

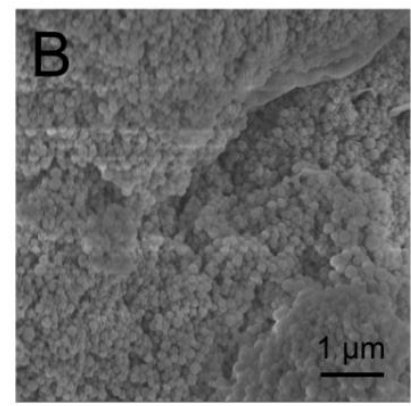

Day 1

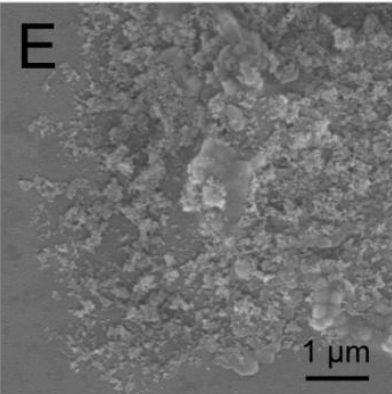

Day 7

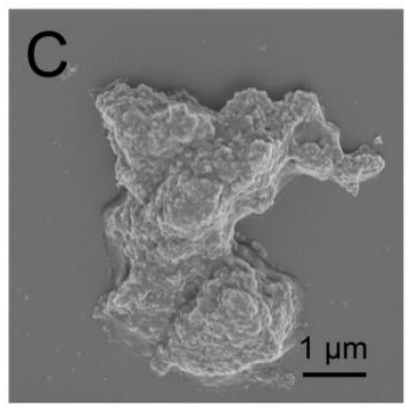

Day 3

Figure S15. The SEM images of HMSNs was placed in NS for different times.
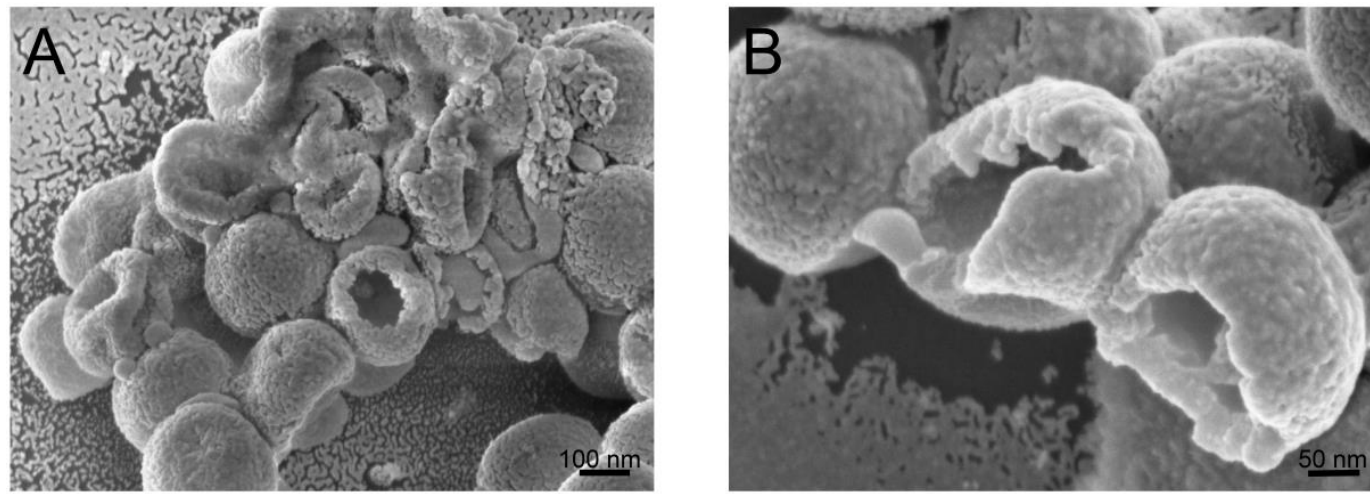

Figure S16. The SEM images of degraded HMSNs of different magnifications. 


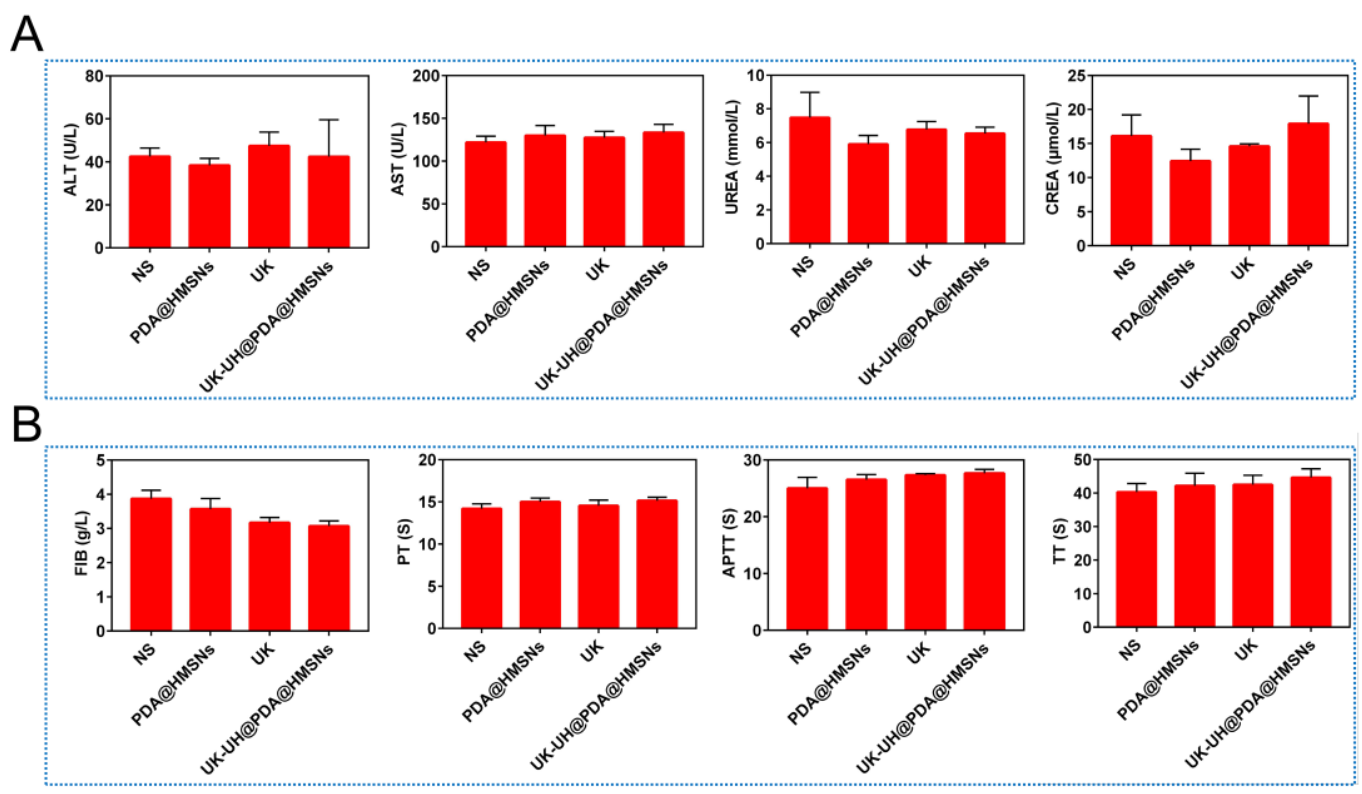

Figure S17. (A) Some indexes of liver and kidney function in SD rats. (B) Four indicators of coagulation in SD rats.

Video S1. UK-UH@PDA@HMSNs was irradiated with near-infrared light in vitro, and continuous microbubble formation was observed.

[1] Hong, S.; Na, Y. S.; Choi, S.; Song, I. T.; Kim, W. Y.; Lee, H. Non-Covalent Self-Assembly and Covalent Polymerization Co-Contribute to Polydopamine Formation. Adv. Funct. Mater. 2012,22, 4711-4717, DOI: 10.1002/adfm.201201156. 


\section{Materials and Methods}

Materials. Urokinase (UK; (5-6) × $\left.10^{4} \mathrm{IU} \mathrm{mg}^{-1}\right)$, Unfractionated heparin (UH; $205 \mathrm{IU}$ $\mathrm{mg}^{-1}$ ), L-menthol (LM), azure A, tetraethyl silicate (TEOS), N-hydroxy succinimide N-hydroxy succinimide (NHS), 1-ethyl-(3-dimethylaminopropyl) carbodiimide (EDC), trimethylol aminomethane (TRIS), cyclohexane (CYH), dopamine hydrochloride (DH), triethanolamine (TEA) and cetyltrimethylammonium chloride (CTAC) were purchased from Macklin (Shanghai, China). Barbiturate standard buffer $\left(\mathrm{pH} 8.6,25^{\circ} \mathrm{C}\right)$ was produced by Haibiao Company (Xiamen, China). ELISA kits were purchased from Jianglai Biological Company (Shanghai, China). Calcein-AM/PI double staining kit and cell count kit-8 (CCK-8) were purchased from Invitrogen Corp (New Mexico, USA) and Everbright Inc (California, USA) respectively. All the reagents were at least analytical grade and used without purification.

Preparation of HMSNs. The preparation of HMSNs was divided into three steps. Firstly, the mixture of $0.8 \mathrm{~mL}$ ammonia, $5.0 \mathrm{~mL}$ water, and $35.7 \mathrm{~mL}$ ethanol was stirred at room temperature for $30 \mathrm{~min}$, and then $1 \mathrm{~mL}$ TEOS was added and stirred for 1 hour. The obtained solution was centrifuged at $8000 \mathrm{rpm}$ for $10 \mathrm{~min}$ and washed with ethanol and deionized water respectively, and then the collected $\mathrm{sSiO}_{2}$ was further dispersed in $20 \mathrm{~mL}$ of deionized water. Subsequently, $6.00 \mathrm{~g}$ CTAC and $0.18 \mathrm{~g}$ TEA dissolved in $40 \mathrm{~mL}$ water were added to a round bottom flask containing $20 \mathrm{~mL} \mathrm{sSiO} 2$ and mixed well, followed by the addition of $2 \mathrm{~mL}$ TEOS and $18 \mathrm{~mL}$ cyclohexane. After mixing, the water and oil phases were delaminated. After stirring 
slowly at $60{ }^{\circ} \mathrm{C}$ for 6 hours, the color of the lower water phase gradually changed into milky white, and then the water phase was collected to obtain $\mathrm{CTAC} / \mathrm{mSiO}_{2} @ \mathrm{SSiO}_{2}$. Subsequently, $30 \mathrm{~mL}$ aqueous phase was diluted with water to $120 \mathrm{~mL}$, and $2.546 \mathrm{~g}$ $\mathrm{Na}_{2} \mathrm{CO}_{3}\left(0.2 \mathrm{M}, 50{ }^{\circ} \mathrm{C}\right)$ was added and stirred violently for 1 hour to obtain hollow structure. After centrifugation and washing at $11000 \mathrm{rpm}$ for $20 \mathrm{~min}$, the CTAC modified the nanoparticles was extracted by ultrasonic extraction of hydrochloric acid/ethanol $(\mathrm{v} / \mathrm{v}=1 / 10)$ solution at least three times to ensure the complete removal of CTAC. Finally, HMSNs was obtained.

Characterization. The morphological characteristics of HMSNs and PDA@HMSNs were determined by scanning electron microscope (SEM; ZEISS/Sigma-300, UK) and transmission electron microscope (TEM; JEM-2100, $200 \mathrm{kV}$, Japan), respectively. The specific surface area and pore diameter of HMSNs were measured by specific surface area and aperture analyzer (Autosor-iQ, USA). The hydrodynamic dimensions and zeta potentials of HMSNs and PDA@HMSNs were measured by particle size and potential tester (Zetasizer Nano ZS-90 instrument, Malvern, UK) at $25{ }^{\circ} \mathrm{C}$. The structural characteristics of HMSNs and PDA@HMSNs were analyzed by Fourier transform infrared spectroscopy (FTIR; Nicolet-5700, USA), X-ray photoelectron spectroscopy (XPS; ESCALAB250Xi, USA) and X-ray diffractometer (XRD; 1611592S, Germany). OD values were detected by multi-function enzyme labeling instrument (15001920, USA). Near infrared laser instrument (1064 nm) was purchased from Hi-Tech Optoelectronics Co. Ltd, China. The absorption spectrum was detected by an ultraviolet-visible spectrophotometer (Shimadzu UV-2600, Japan). All experimental data were repeated at least 3 times.

Drug Loading Rate. In order to determine the drug loading rate of $\mathrm{UH}$, the standard curve of UH was first measured by the azure A dye colorimetric method. Firstly, 0.5, $1.0,1.5,2.0,2.5 \mathrm{~mL}$ standard solution $\left(10 \mathrm{IU} \mathrm{mL}^{-1}\right)$ was added to five centrifuge tubes. Then dropped $1 \mathrm{~mL}$ azure A solution $(0.2 \%, \mathrm{w} / \mathrm{w})$ and barbital hydrochloric acid standard buffer ( $\mathrm{pH} 8.6$ ) into each centrifuge tube in turn, and finally set the total volume to $10 \mathrm{~mL}$ with ultra-pure water. After $30 \mathrm{~min}$ of incubation at $25^{\circ} \mathrm{C}$, the final 
mixture was measured by an ultraviolet spectrophotometer. When drawing the calibration curve, the concentration of standard UH and the absorbance value at 595 $\mathrm{nm}$ were abscissa and ordinate respectively. First, $20 \mathrm{mg}$ of LM powder was added to a $50 \mathrm{~mL}$ beaker, and then $20 \mathrm{~mL}$ of absolute ethanol was added and mixed to form an LM solution. Then, $10 \mathrm{mg}$ UK and $20 \mathrm{mg}$ HMSNs were added to the LM solution. Afterwards, the mixed solution was ultrasonically dispersed and magnetically stirred for overnight. After the ethanol was absolutely evaporated, $80 \mathrm{~mL}$ deionized water was added to the precipitate, and ultrasonically dispersed for $10 \mathrm{~min}$ at room temperature to form a milky stabilized dispersion, then $40 \mathrm{mg} \mathrm{DH}$ was added and stirred for $5 \mathrm{~min}$. The polymerization of dopamine was initiated by adding $20 \mathrm{mg}$ TRIS. After the color of the solution turned into black, the $\mathrm{pH}$ was adjusted to 6.5. The whole reaction was carried out below $30{ }^{\circ} \mathrm{C}$ and the stirring speed was maintained at $300 \mathrm{rpm}$. After the reaction, the product was collected by centrifugation and washed with deionized water for three times. The precipitate was evenly mixed with the UH solution activated by EDC and NHS (5 $\mathrm{g} \mathrm{L}^{-1}, \mathrm{pH}$ 6.5). Finally, UK-UH@PDA@HMSNs could be obtained by stirring the magnetic agitator overnight. The drug loading rate of UH was estimated by measuring the amount of unbound $\mathrm{UH}$ in the supernatant after centrifugation. The drug loading rate of UH was estimated by measuring the amount of unbound $\mathrm{UH}$ in the supernatant after centrifugation. The drug loading was calculated by the following formula:

$$
\text { Drug loading }(\%)=\left(W_{0}-W_{1}\right) / W_{2} \times 100 \% \quad \text { Equation (1) }
$$

$W_{0}$ is the initial weight of the drug, $W_{1}$ is the weight of the drug in the supernatant, and $W_{2}$ is the total mass of drug-loaded nanoparticles. Each sample was tested three times independently.

The content of the UK in UK-UH@PDA@HMSNs was detected by enzyme-linked immunosorbent assay (ELISA). First, $20 \mathrm{mg}$ of LM powder was added to a $50 \mathrm{~mL}$ beaker, and then $20 \mathrm{~mL}$ of absolute ethanol was added and mixed to form an LM solution. Then, $10 \mathrm{mg} \mathrm{UK}$ and $20 \mathrm{mg}$ HMSNs were added to the LM solution, the mixed solution was ultrasonically dispersed and magnetically stirred overnight. After 
anhydrous ethanol volatilization, the precipitate left was added to $30 \mathrm{~mL}$ deionized water. The content of the UK in the supernatant was detected after high-speed centrifugation $(11000 \mathrm{rpm})$. The drug loading rate of the UK was estimated by measuring the amount of unbound UK in the supernatant after high-speed centrifugation. The drug loading rate was defined as the ratio of the drug amount in the final product to the total mass of the input nanoparticles, which could be determined by the purified UK-UH@PDA@HMSNs freeze-dried powder. The drug loading rate could refer to formula (1). Each sample was tested 3 times independently.

Photothermal Properties of PDA@HMSNs. The temperature change was monitored under the irradiation of NIR-II $\left(1064 \mathrm{~nm}, 1.0 \mathrm{~W} \mathrm{~cm}^{-2}\right)$, and the photothermal heating curves of different solutions were obtained. Well-insulated brown vials were used to preserve the solution. The temperatures were recorded using a mercury thermometer.

HMSNs degradation experiment in vitro. A proper amount of HMSNs was added to normal saline (NS), and after ultrasonic dispersion, it was placed in a water bath at $37{ }^{\circ} \mathrm{C}$ for 7 days. Samples were taken on day $0,1,3,5$, and 7 respectively, and the morphology of the samples were photographed by SEM.

Blood Collection and Clotting. The whole blood of healthy New Zealand rabbits was taken by auricular vein puncture. The blood was then put into a test tube without anticoagulants, incubated at $37^{\circ} \mathrm{C}$ for 4 hours, rinsed with NS for 3 times, and dried the surface moisture of the blood clots with filter paper. Finally, the blood clots were divided according to the same size and shape, and their initial weight was recorded.

Evaluation of Thrombolysis in Vitro. First, the effect of UH on thrombolysis of the UK was determined in vitro. The experiment was divided into 5 groups: saline group (I), UH group (II), UK group (III), UK + UH (IV), UK after UH (V). After weighing, the prepared blood clots were put into transparent bottles containing $5 \mathrm{~mL}$ normal saline respectively. After bathing at $37^{\circ} \mathrm{C}$ for 2 hours, the thrombus were taken out, then photoed and weighted at the setting time.

To verify the thrombolytic ability of the UK-UH@PDA@HMSNs in vitro, the 
experiments were divided into four groups: NS + NIR-II group (I), PDA@HMSNs + NIR-II group (II), UK + NIR-II group (III), UK-UH@PDA@HMSNs + NIR-II group (IV). The prepared blood clots were placed in transparent bottles containing $5 \mathrm{~mL}$ saline respectively. The samples were treated with NIR-II $\left(1064 \mathrm{~nm}, 1.0 \mathrm{~W} \mathrm{~cm}^{-2}\right)$ for 2 hours. The thrombolysis was calculated according to the formula: $\Delta W / W_{0} \times$ $100 \% . W_{0}$ is the initial weight of the blood clot and $\Delta W$ represent the weight loss of the blood clot.

Living-Dead Cell Staining Test. HUVECs were evenly inoculated into 24-well plates $\left(5 \times 10^{4}\right.$ cells/well) and cultured in $1 \mathrm{~mL}$ DMEM for 24 hours. Then, the cell culture medium was replaced with $1 \mathrm{~mL}$ solution of different concentrations of PDA@HMSNs $\left(0,10,25,50,100,200 \mu \mathrm{g} \mathrm{mL} \mathrm{m}^{-1}\right)$. After 2 days, the culture medium was removed and the cells were washed with PBS for 3 times. After that, $5 \mu \mathrm{L}$ Calcein-AM/PI was added to the cell sample and incubated in the dark for 15 min. Then the samples were observed by an inverted fluorescence microscope (Olympus Corporation/IX3-CBH, Japan).

Cytotoxicity Assessment. The cytotoxicity of different concentrations of PDA@HMSNs was studied by the CCK-8 assay. HUVECs were inoculated in 96-well microplates $\left(1 \times 10^{4}\right.$ cells/well $)$ overnight. Then HUVECs were co-cultured with different concentrations of PDA@HMSNs $\left(0,5,25,50,100,200 \mu \mathrm{g} \mathrm{mL}^{-1}\right)$ for 12, 24, 48 hours. Then, $10 \mu \mathrm{L}$ CCK- 8 was added and cultured for 2 hours. Finally, the OD value of the sample was measured by the enzyme labeling instrument, and the reference wavelength was 450 nm. The cells without PDA@HMSNs treatment were used as the blank control group, and the survival rate of the untreated group was set at $100 \%$. The cell viability was calculated by the following formula:

$$
\text { Cell viability }(\%)=\left(O D_{450 \mathrm{~nm} / \mathrm{sample}} / O D_{450 \mathrm{~nm} / \mathrm{control}}\right) \times 100 \% \quad \text { Equation }(2)
$$

$O D_{450} \mathrm{~nm} / \mathrm{sample}$ is the absorbance of the experimental group at $450 \mathrm{~nm}$, and $O D_{450}$ $\mathrm{nm} / \mathrm{control}$ is the absorbance of the control group at $450 \mathrm{~nm}$. Each sample was tested three times independently.

Hemolysis Test. The blood of healthy adults was provided by the second Affiliated 
Hospital of Nanchang University and centrifuged (1500 rpm, $15 \mathrm{~min}$ ) with NS for 5 times to obtain pure red blood cells. $0.1 \mathrm{~mL}$ of red blood cells $(20 \%, \mathrm{~m} / \mathrm{m})$ were added to $0.9 \mathrm{~mL}$ ultrapure water, normal saline, and different concentrations of PDA@HMSNs $\left(5,25,50,100,200 \mu \mathrm{g} \mathrm{mL} \mathrm{m}^{-1}\right)$. After incubation at $37^{\circ} \mathrm{C}$ for 1 hour, the whole reactants of each group underwent low-speed (300 rpm, $15 \mathrm{~min})$. Then the supernatant of $0.1 \mathrm{~mL}$ was moved into a 96 -well microplate and the absorbance was measured by microtitration (Synergy 2, Bio-TEK) at the wavelength of $540 \mathrm{~nm}$. The hemolysis was calculated according to the formula:

$$
\text { Hemolysis }(\%)=\left(I-I_{\mathrm{NS}}\right) /\left(I_{0}-I_{\mathrm{NS}}\right) \times 100 \%
$$

$I$ is the absorbance of the supernatant of different concentrations of PDA@HMSNs, $I_{\mathrm{NS}}$ is the absorbance of the supernatant of NS, and $I_{0}$ is the absorbance of complete hemolysis in ultra-pure water.

Biosafety Assessment in Vivo. To evaluate the toxicity and side effects of the nano-drug delivery system, a week after the end of all experiments, the mice were euthanized to collect blood and major organs for further analysis. An automatic biochemical analyzer was used to detect liver, kidney function, and blood coagulation indexes. The main organs and blood vessels were fixed in commercial paraformaldehyde solution, embedded in paraffin, and stained with hematoxylin-eosin (H\&E). Three staining sections were randomly selected and analyzed under a light microscope. 